\title{
Estudios sobre comportamiento electoral, ¿qué explica la participación en las urnas? Un estado del arte*
}

\author{
John Fredy Bedoya Marulanda (Colombia)** \\ Juan Carlos Escobar Escobar (Colombia)*** \\ Aura Sánchez Parra (Colombia) $)^{* * * *}$ \\ Felipe Nieto Palacio (Colombia) ${ }^{* * * *}$
}

\section{Resumen}

El presente artículo presenta un balance de los estudios que analizan la participación electoral, agrupando las dimensiones bajo las cuales se ha estudiado el tema y las evidencias empíricas que las sustentan. El trabajo parte de una comprensión abarcadora que permite evidenciar la complejidad del objeto de estudio, por lo que se enfoca en dar cuenta de la interacción entre las diferentes variables y en exponer los mecanismos explicativos que subyacen a ellas. Esto implica un distanciamiento de análisis convencionales que se concentran en la capacidad predictiva y en la significancia estadística de las variables. Así, se demuestra que no es suficiente abordar la pregunta del comportamiento del elector desde un conjunto de factores separados y se destaca la importancia de la confluencia entre las microvariables y macrovariables a la hora de entender la concurrencia a las urnas.

\section{Palabras clave}

\section{Democracia.}

Comportamiento Político; Elecciones; Participación Política; Voto;

\footnotetext{
* Este artículo es producto del proyecto de investigación Electores urbanos y rurales: una misma competencia, intereses diferenciados. Elecciones para alcalde y presidente, 2003-2014, financiado por la Fundación para la Promoción de la Investigación y la Tecnología (FPIT) del Banco de la República de Colombia y por el Instituto de Estudios Políticos de la Universidad de Antioquia.

${ }^{* *}$ Economista. Magíster en Ciencia Política. Grupo Estudios Políticos, Instituto de Estudios Políticos, Universidad de Antioquia UdeA. Calle 70 No. 52-21, Medellín, Colombia. Correo electrónico: john. bedoyam@udea.edu.co Orcid: https://orcid.org/0000-0002-5616-4616

*** Sociólogo. Magíster en Ciencia Política. Grupo Estudios Políticos, Instituto de Estudios Políticos, Universidad de Antioquia UdeA. Calle 70 No. 52-21, Medellín, Colombia. Correo electrónico: juan. escobare@udea.edu.co Orcid: https://orcid.org/0000-0002-1073-0109

**** Politóloga. Grupo Estudios Políticos, Instituto de Estudios Políticos, Universidad de Antioquia UdeA. Calle 70 No. 52-21, Medellín, Colombia. Correo electrónico: auram.sanchezp@gmail.com

***** Politólogo. Correo electrónico: felipenieto8@gmail.com
} 


\title{
Cómo citar este artículo
}

Bedoya Marulanda, John Fredy; Escobar Escobar, Juan Carlos; Sánchez Parra, Aura y Nieto Palacio, Felipe. (2019). Estudios sobre comportamiento electoral, ¿qué explica la participación en las urnas? Un estado del arte. Estudios Políticos (Universidad de Antioquia), 54, pp. 177-198. http://doi.org/10.17533/udea.espo. n54a09

\section{Electoral Behavior Studies. What Explains the Turnout? State of the Art}

\begin{abstract}
This article presents a balance of the studies that analyze the electoral participation, grouping the dimensions under which the subject has been studied and the empirical evidences that sustain them. The work is based on a comprehensive understanding of the object of study that allows to show its complexity. The study focuses on accounting for the interaction between the different variables and on revealing their explanatory mechanisms. This implies a separation from conventional analyzes that focus on the predictive capacity and the statistical significance of the variables. Thus, the article concludes that it is not enough to address electoral behavior from a set of separate factors, and highlights the importance of the

[178 ] confluence between the micro and macro variables when it comes to understanding the turnout of voters.
\end{abstract}

\section{Keywords}

Political Behavior; Elections; Political Participation; Vote; Democracy. 
Estudios sobre comportamiento electoral, ¿qué explica la participación en las urnas?...

\section{Introducción}

El estudio del comportamiento electoral ha concentrado sus esfuerzos en tratar de entender la forma en que los electores toman las decisiones, en especial la de asistir a las urnas. En un inicio, el análisis partió de razonar en cómo la identificación subjetiva y la identidad grupal moldean el comportamiento electoral (Lazarsfeld, Berelson y Gaudet, 1968), tomando en cuenta características socioeconómicas de los votantes como los ingresos, el nivel educativo, la edad, el sexo, la religión, entre otros. Contribuciones posteriores se encargaron de estudiar las construcciones mentales que los individuos tienen sobre los elementos políticos, incluyendo los valores, las creencias, las actitudes y los sentimientos (Campbell, Converse, Miller y Stokes, 1980). Una visión panorámica sobre los primeros avances de estos postulados puede verse en Carmen Pérez Baralt (2006).

Paralelamente, con los planteamientos de Anthony Downs (1957), se desarrolla una nueva línea de pensamiento que homogeniza este tipo de investigaciones y pone como punto de enfoque la efectividad del voto. Este término, de manera genérica, es entendido como la probabilidad de que un voto pueda hacer la diferencia en los resultados de una elección y que ello permita a un ciudadano adquirir los beneficios de las promesas hechas por su candidato; es decir, el ciudadano valora la probabilidad de que su voto sea realmente efectivo y, dado ese resultado, evalúa si amerita incurrir en los costos de votar. En este escenario, el resultado final será siempre una abstención generalizada, ya que los costos serán apreciados como mayores en comparación con los beneficios, generando un alto incentivo a tomar la conducta de un free rider (Contreras y Navia, 2013).

Este postulado ha sido ampliamente debatido dado que una abstención generalizada no es lo que señala la evidencia empírica. En este sentido, la literatura política contemporánea ha complementado este tipo de análisis aduciendo que los votantes pueden buscar otros referentes para actuar, más allá de la lógica racional de costo-beneficio (Wang, 2013). De allí que exista la posibilidad de que la decisión de votar sea un entramado compuesto por macrovariables, como el tamaño de la población, el diseño institucional, las dinámicas de la competencia; y microvariables, como el deber cívico, la eficacia política, las redes de comunicación, la integración social, entre otras. 
A propósito de lo anterior, este artículo recoge, a manera de estado del arte, otras explicaciones, más allá de los postulados canónicos, ${ }^{1}$ con las cuales se ha intentado responder la pregunta de por qué la gente vota. La intención de resaltar otros enfoques en el estudio de la participación electoral radica en visibilizar aquellas variables, que a lo largo del desarrollo de este subcampo de investigación han demostrado que la explicación de la participación electoral no puede reducirse al uso exclusivo y aislado de variables como las socioeconómicas —edad, sexo, género, educación, entre otras—, las psicológicas — voto emocional, expresivo o de protesta, identidad partidista, entre otras - y la elección racional —cálculo costo-beneficio-, sino que debe comprender la interacción entre múltiples factores que logren dar cuenta de la complejidad de este objeto de estudio (Magalhães, Segatti y Shi, 2015).

Este artículo es relevante por varios motivos. En primer lugar, porque a pesar de que la participación electoral es uno de los temas más tratados por la Ciencia Política todavía se identifican obstáculos para explicar el grado de afluencia a las urnas, puesto que el efecto de las variables usadas hasta el momento presenta contradicciones de un contexto a otro (Dalton y Anderson, 2010). En segundo lugar, se hace énfasis en los mecanismos explicativos, de tal manera que se sustenta teóricamente la relación entre las distintas variables independientes y la participación electoral, denotando la complejidad del fenómeno y diferenciándose de otros estados del arte que han hecho más énfasis sobre la capacidad predictiva y significancia estadística de las variables (Geys, 2006; Cancela y Geys, 2016). Finalmente, el artículo, como una actualización y consolidación bibliográfica de los estudios del comportamiento del elector, servirá como referente para futuros estudios que se desarrollan en este ámbito en América Latina, los cuales acuden, recurrentemente, a autores clásicos (Bedoya, 2015).

El lector se encontrará con el siguiente esquema expositivo. En primer lugar, se examina la literatura centrada en las variables institucionales que han ayudado a responder la pregunta mencionada. Asuntos como la proporcionalidad del sistema, el número de partidos, la obligatoriedad del voto, la simultaneidad de las elecciones, entre otros,

\footnotetext{
${ }^{1}$ Con la idea de ilustrar suficientemente el efecto de las distintas explicaciones que se le ha dado a la decisión de votar, en este artículo se excluyen las variables que han sido revisadas y agrupadas en factores sociodemográficos, como lo hacen Kaat Smets y Carolien Van Ham (2013).
} 
son usados a menudo para explicar, a partir de enfoques institucionales, la concurrencia a las urnas.

El segundo grupo de explicaciones halladas en los textos se han centrado en observar las dinámicas de la competencia electoral, especialmente en variables relacionadas con las campañas y lo reñido de las elecciones. A diferencia de las variables institucionales, se trata en este caso de factores de corto plazo que pueden influir en la decisión de un elector de acudir a las urnas.

La tercera fuente de explicación al problema planteado guarda relación con el apego de los ciudadanos a su territorio y el interés colectivo por los asuntos políticos, sociales y económicos que le interesan porque los consideran propios y cercanos. En este caso, se trata de aproximaciones más cercanas a una perspectiva sociológica, dado que involucran temas de comunidad, adhesión a un grupo social, heterogeneidad, entre otros.

El cuarto y último apartado identifica, en el corpus de textos escogido, tres fuentes mediante las cuales los electores adquieren la información necesaria para acudir a votar el día de las elecciones: los partidos, los medios y la socialización política.

\section{Variables institucionales para explicar la participación electoral}

En los estudios electorales, el diseño del sistema electoral y de partidos ha contribuido significativamente a la explicación del comportamiento del votante. La proporcionalidad del sistema, la obligatoriedad del voto, la simultaneidad de las elecciones, las leyes de registro, la fatiga electoral y el carácter bicameral o unicameral del parlamento son las variables más recurrentes - a partir de una perspectiva general - para entender la participación de manera comparada y desde una visión institucional.

En primer lugar, la variable institucional a la que mayor peso se le ha atribuido es al diseño de representación que un sistema posee. Al respecto, la literatura muestra que la participación electoral es más alta en sistemas con representación proporcional que en sistemas de tipo mayoritario (Gaarsted, 2002; Jackman y Miller, 1995; Boulding y Brown, 2013), debido a que en estos últimos los votantes pueden percibir que desperdician su voto si un 
candidato tiene una alta posibilidad de ganar las elecciones, mientras que en los sistemas proporcionales, donde hay más escaños por distribuir, todos los partidos tienen posibilidades de ocupar alguno de ellos, incluso los partidos más pequeños.

El estudio realizado por Bernard Gofman y Peter Selb (2010) es quizá uno de los más ilustrativos en torno a la relación entre los sistemas electorales y la participación. Los autores concluyen que, para España y Suiza, las circunscripciones plurinominales en sistemas proporcionales exhiben mayores tasas de participación que las circunscripciones uninominales en sistemas desproporcionales. Algo similar encuentran Markus Freitag (2010), para el caso de los cantones suizos, Annie Gaarsted (2002), en las elecciones locales de los países europeos, y Robert Jackman y Ross Miller (1995), en veintidós democracias industrializadas en el mundo.

Lo mismo puede decirse de las fórmulas electorales para la repartición de escaños. Aquellas que son más proporcionales dan incentivos tanto a partidos como a votantes para una mayor movilización (Jackman y Miller, 1995; Kuenzi y Lambright, 2007; Stockemer, LaMontagne y Scruggs, 2012), ya que facilita la emergencia de nuevos partidos y amplía la gama de opciones [182 ] que poseen los votantes entre las cuales pueden elegir (Gofman y Selb, 2010; Henderson y McEwen, 2010). Al respecto, Carew Boulding y David Brown (2013) encuentran que para las elecciones en el ámbito municipal de Bolivia, cuyas normas de repartición de escaños son proporcionales, la participación electoral es mayor, en comparación con el caso de Brasil, cuyas elecciones locales se rigen por reglas que favorecen a los partidos mayoritarios, lo que conlleva a que decrezca la participación en la medida en que aumenta el número de partidos. Esto se corrobora en el estudio de Michelle Kuenzi y Gina Lambright (2007), quienes analizan 32 elecciones legislativas en África, en donde el promedio de participación de los sistemas más proporcionales es de $71 \%$, en comparación con $59 \%$ para las elecciones realizadas con reglas mayoritarias.

Relacionado con lo anterior, también se encuentra en la literatura estudios que se preguntan por la incidencia de la fragmentación del sistema de partidos sobre las tasas participación electoral (Blais, 2006; Geys, 2006; Kuenzi y Lambright, 2007; Boulding y Brown, 2013). En primer lugar, está el argumento que sostiene que el multipartidismo tiene una relación negativa con la concurrencia a las urnas, ya que mientras más partidos estén en 
competencia hay más propuestas por las que se deben indagar; además, la existencia de múltiples partidos tiende a afectar negativamente el efecto del voto de una persona sobre los resultados, ya que las posibilidades de traducir sus votos en la formación de un gobierno se ven reducidas por la impredecibilidad que supone la creación de coaliciones en un escenario tan heterogéneo. En segundo lugar, se ha planteado que la fragmentación partidista puede aumentar la asistencia a las elecciones, ya que los partidos pueden concentrarse en un número limitado de asuntos y se puede identificar una línea programática de manera más clara y fácil para el votante, enriqueciendo las posibilidades de que un votante se sienta representado por algún partido.

Los resultados empíricos hallados se decantan principalmente en la primera línea. En su trabajo sobre los sistemas multipartidistas del África subsahariana, Kuenzi y Lambright (2007) mencionan aquellos estudios que han encontrado que el multipartidismo tiende a disminuir la concurrencia a las urnas. Exponen que Siaroff y Merer concluyen que existe una relación positiva entre sistemas bipartidistas y la participación en las elecciones legislativas en Europa desde 1990; de manera complementaria, las autoras expresan que Kostadinova apunta hacia una relación negativa entre la participación y el multipartidismo en la Europa poscomunista. Lo mismo aplica para el estudio de Freitag (2010) en las elecciones parlamentarias suizas.

Otra de las variables institucionales tiene que ver con la estructura del Parlamento. Tanto para Jackman y Miller (1995), como para Carolina Fornos, Timothy Power y James Garand (2004), en los sistemas unicamerales la participación electoral es más alta, pues los votantes perciben que las elecciones adquieren mayor importancia debido a que los actos legislativos poseen menos trámites y, por tanto, su voto incidirá más en los resultados; es decir, el unicameralismo es fundamental para producir gobiernos decisivos y se salta la engorrosa tarea de hacer compromisos con otras instancias de gobierno.

En segundo lugar, otro grupo de variables institucionales que han sido evaluadas empíricamente da cuenta de la logística del evento electoral. Algunos estudios afirman que la existencia del voto obligatorio o de sanciones para aquellos que se abstienen contribuyen a incrementar la participación electoral, ya que aumentan los costos de no asistir a las urnas y pueden reforzar la presión social que tienen los ciudadanos para hacerlo (Geys, 2006; Gaarsted, 2002). Freitag (2010) demuestra lo anterior al concluir 
que en cantones como Schaffhaunse, Suiza, el promedio de participación supera por casi $20 \%$ a aquellos cantones donde el voto es voluntario. En el caso latinoamericano, la obligatoriedad del voto es una variable central. La literatura comparada demuestra que los niveles de participación son más altos en los países que tiene voto obligatorio (Fornos, Power y Garand, 2004). Esto es evidente en Brasil y Perú, los cuales son países que, con voto obligatorio, tienen niveles de participación superiores a países como México o Colombia, donde el voto es voluntario. Incluso, los estudios comparativos en un mismo país demuestran que los cambios en la obligatoriedad del voto tienen efectos significativos en la concurrencia a las urnas, como lo muestra el caso chileno (Mackena, 2015).

Dentro las variables relacionadas con la logística electoral también se pueden encontrar las leyes que rigen el registro de los votantes. Tal y como lo señalan Fornos, Power y Garand (2004), en los países latinoamericanos, donde la reglamentación sobre la inscripción es más flexible, la concurrencia a las urnas es mayor, dejando como explicación a este fenómeno el bajo costo que representa para los votantes la inscripción.

La frecuencia con la que los individuos son llamados a elegir sus dirigentes [184 ] también afecta la variable de estudio, pues el ciudadano es propenso a sufrir de fatiga electoral cuando el tiempo entre una elección y otra es menor (Boyd, 1986). Así lo demuestran Jackman y Miller (1995) al sugerir que la elevada frecuencia con la que los ciudadanos son llamados a votar en países como Suiza y Estados Unidos influye negativamente en la participación electoral. Lo mismo sustenta Richard Boyd (1986), quien determina que los bajos niveles de participación en el sur de Estados Unidos obedecen al complejo calendario electoral.

En esta misma vía, la tasa de participación electoral aumenta en aquellos países donde las elecciones locales y las nacionales son llevadas a cabo el mismo día (Caren, 2007; Hajnal y Lewis, 2003). Por ejemplo, Neal Caren (2007), en un estudio realizado sobre las 322 elecciones de 38 ciudades en Estados Unidos, concluye que aquellos comicios en que coincidieron las elecciones presidenciales y locales presentaron un índice de concurrencia electoral $27 \%$ mayor que en las demás ciudades. Lo mismo sucede con los hallazgos de Zoltan Hajnal y Paul Lewis (2003) para las distintas ciudades de California, con los de Irene Delgado (1998) en los municipios españoles, con los de Fornos, Power y Garand (2004) para los países latinoamericanos 
y con los de Kuenzi y Lambright (2007) en los procesos electorales en África para las elecciones presidenciales y legislativas. Esto lo explica Benny Geys (2006), aduciendo que la simultaneidad permite que los medios se interesen más en las elecciones locales reduciendo los costos de votación, asunto que se trata más adelante.

\section{Dinámicas de la competencia electoral y sus efectos sobre la concurrencia en las urnas}

Entre los estudios sobre la participación electoral, la manera en que se desarrollan las elecciones se ha destacado como uno de los factores más relevantes. En este caso, las macrovariables más significativas son aquellas que se presentan en el corto plazo o que varían entre comicios, tales como lo reñido de las elecciones, el gasto en campaña y el uso de campañas negativas. También se ha hecho uso de elementos analíticos de orden individual micro- para explicar cómo el fenómeno de la percepción que tienen los ciudadanos sobre la importancia de unas elecciones y lo que se encuentra en juego en estas afectan su asistencia a las urnas. Empero, en los mecanismos explicativos de cada una de las variables enunciadas se establecen vínculos con otras, que pasan a ser intervinientes y alteran su efecto, entre ellas el tipo de elección — de primer o segundo orden—, el tamaño de la población y la movilización partidista.

Con relación a las macrovariables, ligadas a las dinámicas electorales que incluyen lo reñido de las elecciones, se pueden mencionar dos vertientes: en la primera, la relación indica que entre más reñida es una elección mayor es el nivel de concurrencia a las urnas (Cancela y Geys, 2016; Blais, 2006; Caren, 2007). Este tipo de análisis se ha expandido y la hipótesis se ha probado en otros escenarios, como es el caso de António Tavares y Jered Carr (2012) en las elecciones locales de Estados Unidos, y de Irvin Soto y Willy Cortez (2014) en México. Si bien en estos estudios no se no distinguen las variables intervinientes en la relación planteada, conviene decir que la movilización partidista ha sido utilizada como argumento para explicar la influencia positiva de la competitividad sobre participación electoral. Esto porque las elecciones reñidas conducen a una mayor actividad de los partidos y de sus élites políticas, produciendo un incremento en los esfuerzos de campaña —incluyendo el gasto en la misma - y una alta visibilidad de la coyuntura electoral, situación que atrae a los electores (Geys, 2006). 
Otro de los mecanismos explicativos para entender la relación entre unas elecciones reñidas y la asistencia a las urnas es que unos comicios competitivos despiertan el interés de los votantes en la campaña, ya que les proporciona la idea de que su voto puede resultar determinante en el resultado y, por tanto, se interesan más en el proceso (Gaarsted, 2002; Tavares y Carr, 2012; Soto y Cortez, 2014). Serguei Kaniovski y Dennis Mueller (2006) explican que los electores no concurrirán a las urnas en medio de unas elecciones poco competitivas, para no desperdiciar su voto en candidatos o partidos sin oportunidades de ganar (Lago y Costa, 2014). Sin embargo, Aaron Edlin, Andrew Gelman y Noah Kaplan (2007) —siguiendo a Downs (1957)_ refutan esta idea, advirtiendo que la probabilidad de que un voto sea decisivo es minúscula, pero como lo plantean Sandra Breux, Jérôme Couture y Nicole Goodman (2016), el efecto de unas elecciones reñidas sobre la participación electoral es significativa en poblaciones pequeñas.

La otra vertiente sustenta que la relación entre la participación electoral y lo reñido de las elecciones es nula o inexistente. Tatiana Kostadinova y Timothy Power (2007) no hallan ningún efecto del margen de victoria entre los candidatos y la asistencia a los comicios en Europa del Este y América Latina entre 1978 y 2003, resultado que es compartido por Fornos, Power y Garand (2004). Por su lado, Daniel Stockemer, Bernadette LaMontagne y Lyle Scruggs (2012), al indagar el nexo entre la corrupción y la participación electoral en todas las democracias conocidas, adoptaron entre sus variables de control el grado de competitividad de las elecciones, concluyendo que esta no guarda relación estadísticamente significativa en su estudio. Asimismo, en su examen de las elecciones primarias y generales en Estados Unidos, Anthony Nownes (1992) no derivó un nexo entre las percepciones que los votantes tienen sobre lo reñido de las campañas y la decisión individual de votar. Este mismo desenlace se puede rastrear en Carmen Ortega y José Trujillo (2013) para las elecciones locales de Andalucía en 2011.

El gasto en campañas también ha sido una fuente para los análisis sobre las dinámicas de la competencia y la participación electoral. A partir de su metaanálisis, Geys (2006) sostiene que los trabajos empíricos apoyan la afirmación de que existe una relación positiva entre ambas variables; no obstante, encuentra que el efecto de los gastos en campaña puede estar vinculado a otros elementos, como los mensajes que son desplegados por los candidatos y partidos, y el ámbito en el que se desarrollan las elecciones, 
siendo más efectivo en los comicios nacionales que en los subnacionales (Cancela y Geys, 2016). Sin embargo, esta hipótesis no se corrobora en todos los casos, pues Octaviano Moya (2007), al analizar las elecciones presidenciales de 2006 en México, encontró que quien menos gastó fue quien obtuvo la victoria.

En relación con las campañas negativas, Geys (2006) expone dos líneas argumentativas: en la primera, los anuncios negativos pueden resultar dañinos para la imagen del candidato atacado y del candidato que profiere el ataque, disminuyendo el apoyo electoral de ambos y reduciendo la participación electoral; de la misma manera, este tipo de campañas desincentivan la concurrencia a las urnas al generar una visión adversa de la política en general. En la segunda línea argumentativa explica que las campañas negativas pueden llegar a incrementar la participación electoral, debido a que se genera una gran carga de información sobre el rendimiento de los candidatos, aspecto que disminuye el costo de información para el votante, construye una imagen definida de los candidatos y tiende a producir respuestas afectivas entre los ciudadanos.

Con respecto a las microvariables, dentro de las dinámicas de la competencia y la participación electoral se ha analizado el comportamiento de los votantes cuando estos estiman que en una elección hay una apuesta electoral —stakes - muy alta, según la definición de sus necesidades e intereses políticos. Considerando esto, Jørgen Andersen, Jon Fiva y Gile Natavik (2014) encuentran, para el caso de Noruega, que en las localidades donde hay mayores ingresos y, en consecuencia, hay más en juego, los electores circunscritos a ellas tienden a enfocarse en la política local, aumentando la participación electoral para este ámbito. Contrario a dicho hallazgo, Jonas Lefeve y Peter Van Aelst (2014) concluyen que las apuestas electorales son consideradas como superiores en las elecciones de primer orden —nacionales-.

Finalmente, es importante mencionar el efecto del interés en las campañas sobre la participación electoral. Nownes (1992), a partir de una encuesta diseñada para medir la disposición ciudadana a seguir el desarrollo de toda la campaña presidencial en Estados Unidos, encontró que la atención prestada a los comicios incrementa las posibilidades de que el votante asista a depositar su voto. 


\section{Tamaño y homogeneidad de la población}

Dentro de las perspectivas macroanalíticas, el tamaño de la población es central para explicar las diferencias en la concurrencia a las urnas. El análisis de esta variable parte de considerar que la participación es, en buena medida, resultado de la integración social y de los lazos de identificación que son más fuertes en comunidades pequeñas. Lo mismo se ha dicho para explicar el efecto que tienen el nivel de heterogeneidad u homogeneidad del grupo social sobre la explicación de la participación electoral.

Adicionalmente, hay que mencionar que estas variables de nivel agregado han sido abordadas a partir de explicaciones micro, imputando que el apego de los ciudadanos a su territorio o grupo social despierta el interés individual por los asuntos políticos, sociales y económicos que le compete a su comunidad, es decir, incentiva el deber cívico. Así, la adhesión de los individuos a un grupo social y la fuerza con la que se establece esta parece tener incidencia positiva sobre la concurrencia electoral (Ben-Bassat y Dahan, 2012; Zimmer, 1983).

De esta manera, comunidades más pequeñas tienden a asistir más a las [188] urnas en comparación con grupos sociales más grandes (Kaniovski y Muller, 2006). Esto obedece a que en las poblaciones más pequeñas se establecen vínculos de cercanía con las instituciones locales y líderes municipales, lo que permite a los ciudadanos realizar demandas de manera directa a los representantes o candidatos sobre sus problemas; además, los vecinos en áreas geográficas más cercanas son más proclives a entrar en contacto, compartir preocupaciones y organizar la acción cívica (Morlan, 1984; Tavares y Carr, 2012; Delgado, 1998; Ortega y Trujillo, 2013; Jácome, 2013).

Es importante mencionar que la proximidad gestada bajo el seno de poblaciones de tamaño reducido tiene incidencia especialmente con el gobierno local, en tanto es en dicho escenario donde la comunidad enmarca su proceso de empoderamiento y donde comienza a confiar en esta institución (Hajnal y Lewis, 2003), permitiendo a las élites locales y regionales fomentar el desarrollo de identidades, debido a la experiencia de las estructuras políticas autónomas, aumentando así el interés en las elecciones (Blais, Anduiza y Gallego, 2011; Henderson y McEwen, 2010).

Asimismo, las poblaciones pequeñas se caracterizan por la creación de fuertes redes sociales y culturales de control que estimulan la participación. Al 
compartir valores y creencias comunes, los ciudadanos refuerzan su sentimiento e identidad con la comunidad que habitan, lo que motiva el apoyo y la defensa de los intereses comunales (Gaarsted, 2002). Esto significa que cuando los individuos se sienten atados a los principios que rigen las dinámicas de la comunidad el acto de votar se percibe como una manifestación del deber cívico — el cual es un factor político de orden individual — en virtud de las características sociales de esta, por lo que no votar puede causar sanción social por parte de los coterráneos (Funk, 2008; Galais y Blais, 2015) y la pérdida de prestigio o reputación individual (Geys, 2006; Riker y Ordeshook, 1968).

Entre las corroboraciones empíricas de estos postulados, Ortega y Trujillo (2013) analizan las elecciones locales en Andalucía y concluyen que el tamaño de la población es un factor explicativo con efecto negativo sobre la conducta electoral. Esto ocurre porque en las poblaciones más pequeñas se refuerzan los dispositivos de control social entre sus ciudadanos, así los electores asisten a las urnas condicionados por la presión ejercida por la comunidad. Los mismos hallazgos tiene Gaarsted (2002) en la comparación entre Dinamarca, Holanda, Noruega, Suiza y Reino Unido para las elecciones municipales.

Igualmente, Caren (2007) se preocupa por entablar una relación entre la participación electoral y el tamaño de la población; sin embargo, explica que es el crecimiento poblacional la variable que realmente tiene incidencia sobre la concurrencia de los ciudadanos en las urnas. Tras analizar 322 elecciones entre 1979 y 2003 en 38 ciudades norteamericanas, concluye que las ciudades con bajos niveles de crecimiento poblacional presentan tasas más altas de participación en comparación con aquellas que crecen más rápido. Un posible mecanismo explicativo de esto es la pérdida de sentido comunitario que se acentúa cada vez que una población crece.

Con relación al efecto del tamaño de la población sobre la concurrencia a las elecciones nacionales o locales, Ailsa Henderson y Nicola McEwen (2010) analizan las elecciones regionales de nueve países para concluir que las tasas de participación electoral son más altas en zonas de mayor apego regional. Desde la perspectiva del electorado se puede elevar la importancia política de los asuntos subnacionales y así aumentar los intereses que conllevan al ciudadano a votar. Esto es corroborado por Carol Galais y André Blais (2015), quienes al comparar ocho regiones de países como Alemania, España y Suiza también concluyen que el mayor apego a la región conduce a tener un sentido más fuerte del deber en las elecciones. 
Por otra parte, en conjunción con el tamaño de la población, el nivel de heterogeneidad $\mathrm{u}$ homogeneidad del grupo social también cumple su papel para la explicación de la participación electoral. Cuando los individuos tienen diferentes preferencias debido a un alto grado de heterogeneidad, tal y como se presenta en grandes ciudades, la participación en actividades sociales es más exigua. En ese sentido, mientras el nivel de heterogeneidad sea menor, la solidaridad y el vínculo social será más fuerte, lo que conlleva a un mayor interés en la participación de actividades políticas (Lago, Bermúdez, Guinjoan y Simón, 2014; Henderson y McEwen, 2010).

Por ejemplo, Avi Ben-Bassat y Momi Dahan (2012) muestran, para las elecciones locales en Israel, que los ciudadanos son más proclives a votar si los candidatos son miembros de la comunidad étnica a la que hacen parte. De esta manera, los autores concluyen que la participación electoral en localidades pequeñas y árabes es mayor en comparación a los resultados presentados en los pueblos mayoritariamente judíos, pues las primeras se componen de miembros de la comunidad Humala, un grupo de familias con antepasados comunes.

También, Ignacio Lago, Sandra Bermúdez, Marc Guinjoan y Pablo [190 ] Simón (2014), en el marco de las elecciones de la Cámara baja legislativa de veintidós países, demuestran que la participación electoral está relacionada negativamente con la fragmentación étnica y lingüística, tal y como se espera teóricamente. Mientras más diverso sea un grupo en términos de su idioma y sus características étnicas la tasa de asistencia a las urnas será más baja. Esto ocurre debido a que la heterogeneidad trae consigo la existencia de preferencias políticas disímiles. Cuando los individuos discrepan significativamente con respecto a sus inclinaciones tienden a participar menos en actividades sociales y políticas, lo que se traduce así en niveles más bajos de votación.

Igualmente, Giogio Bellettini, Carlotta Berti y Chiara Monfardini (2014) encuentran que la proporción de inmigrantes, como indicador de la heterogeneidad, está asociada negativamente con la participación electoral en las elecciones locales italianas entre 2004 y 2009. Kaniovski y Mueller (2006) también confirman la relación negativa entre el tamaño de la población, heterogeneidad y participación electoral, al examinar el caso de un referendo sobre el idioma que debía ser usado en los distritos escolares noruegos, los autores resuelven que el tamaño de la comunidad y la heterogeneidad lingüística tienen un efecto negativo sobre la concurrencia electoral. 


\section{Comunicación social y política en el proceso electoral}

Los medios de comunicación desempeñan un papel fundamental en el proceso de participación política y electoral de los votantes, ya que el grado de dificultad para acceder a la información genera mayor o menor grado de atracción a votar. En este sentido, estudiado desde el ámbito individual, se ha señalado que el costo de la información y la participación electoral guardan una relación negativa, por lo que se entiende que entre mayor sea el costo de obtener información en torno a las votaciones y a las propuestas de los candidatos menor será la concurrencia a las urnas por parte de los ciudadanos.

La literatura sobre los medios de comunicación y la concurrencia a las urnas han sido clasificadas dentro de los análisis de tipo individual que se centran de manera específica en el elector; sin embargo, los trabajos empíricos en esta área presentan referencias tanto a racionalizaciones individuales como a nivel agregado — descentralización, el tipo de elección y hasta el nivel de modernización-, todo esto con el fin de poder otorgar explicaciones que consideren diversos matices en sus resultados.

Sobre este asunto, Rune Sørensen (2016) apunta a que el impacto de los medios se produce en tres direcciones principales: a) la falta de información sobre el desempeño de los gobiernos puede conducir a una baja participación electoral, aspecto que puede llevar a que el gobierno de turno sirva a sus propios intereses al no tener una vigilancia ciudadana activa; b) la baja participación electoral tiende a desprestigiar la representación política con implicaciones en la toma de decisiones por parte del gobierno; c) los gobiernos pueden discriminar a los ciudadanos que viven en zonas con acceso limitado a los medios de comunicación, ya que carecen de suficiente información sobre las políticas.

Por otro lado, al momento de medir la magnitud con la que los medios de comunicación afectan a los ciudadanos, se han encontrado dos tendencias predominantes: la primera se encuentra acuñada en la teoría hipodérmica, la cual sostiene que los mensajes emitidos por las campañas electorales y los medios de comunicación penetran en los electores después de una evaluación mínima del mensaje, cambiando sus opiniones e influyendo en su voto; la segunda tendencia está soportada en la teoría de los efectos mínimos, que plantea que los medios y las campañas tienden a reforzar lo que sería su voto normal, por lo que el votante interioriza la información que apoya su decisión (Moya, 2007). 
En este orden de ideas, se ha encontrado que la exposición a los medios tiende a incrementar el deseo y la habilidad individual de votar. El trabajo de Sørensen (2016) apunta en esta dirección, al mirar el efecto que tuvo la introducción de la televisión en la participación electoral noruega a partir de datos del ámbito municipal. A diferencia de los resultados obtenidos por este tipo de análisis en Estados Unidos, el autor encuentra que la participación electoral aumenta con la llegada de la televisión, ya que hay una mayor cobertura de las campañas electorales, lo que a su vez incrementa el compromiso e interés del ciudadano con la política. El autor destaca que la diferencia con los resultados sobre la concurrencia a las urnas de los estadounidenses puede estar vinculada con el tipo de información ofrecida en los medios de dicho país, por lo que aclara que el efecto de la televisión parece estar ligado al tipo de mensaje que se transmite a los votantes.

Por su parte, Kuenzi y Lambright (2007), si bien se encargan de observar la exposición a los medios como un factor de corte individual para comprender la concurrencia a las urnas en el África subsahariana, se valen de variables de corte agregado ligadas a la modernización para realizar su medición. En este sentido, el impacto que tiene la exposición a los medios de comunicación fue medida a través del número de radios per cápita por [192] país, ya que las tasas de alfabetización no son lo suficientemente altas en la región y los periódicos tienden a ser relativamente costosos. Los resultados evidenciaron una relación positiva entre el número de radios per cápita y la participación electoral como producto de la información que se divulga sobre las elecciones, su logística y tiempos.

Lo anterior da cuenta de la importancia que tiene la cobertura que los medios de comunicación le proporcionan a una elección. En este orden ideas, se ha identificado que los medios proporcionan mayor importancia a las elecciones nacionales, ya que las incluyen de manera activa en sus agendas. A partir de la observación de las elecciones locales, nacionales y del Parlamento Europeo en los Países Bajos entre 2009 y 2010, Lefeve y Van Aelst (2014) demuestran que los partidos, los votantes y los medios dan mayor espacio a las elecciones de primer orden — nacional — que a las de segundo orden —supranacionales y locales—, situación que se vincula al poder que detenta la institución o cargo por el que se compite en las elecciones (Blais, Anduiza y Gallego, 2011).

Se ha registrado que los medios invierten más en las elecciones nacionales debido a que consideran que en ellas hay más en juego stakes- que en las locales (Henderson y McEwen, 2010), por lo que es 
posible afirmar que los votantes urbanos tienen más información política de los medios y están más propensos a votar (Fornos, Power y Garand, 2004). Del planteamiento anterior se desprende una carencia en el cubrimiento de las elecciones locales, por lo que los votantes de este ámbito deben esforzarse más para adquirir información. Sin embargo, André Blais, Eva Anduiza y Aina Gallego (2011) resaltan que la descentralización es un factor que ha llevado a que existan medios de comunicación masiva regionales que trabajan en torno a temas de la política local, cubriendo candidatos y partidos más visibles, lo que contribuye al desarrollo de preferencias electorales de los votantes en lo subnacional.

\section{Otras variables}

Es importante llamar la atención sobre otras variables que aquí no se abarcan por su ausencia en la literatura revisada y que son necesarias al momento de estudiar un fenómeno tan complejo como la participación electoral. Entre estos, procesos históricos como la relación entre partidos y votantes basadas en redes de clientela, la intermediación política, así como los continuos cambios normativos que regulan la competencia partidista y la administración estatal como la descentralización (Blais, Singh y Dumitrescu, 2014; Blais, Anduiza y Gallego, 2011) se erigen como factores que, en conjunto, influyen en el imaginario que los ciudadanos poseen sobre el funcionamiento de este proceso democrático.

Adicional a ello, no debe dejarse pasar de largo asuntos como la estabilidad y la calidad de la democracia, y las dinámicas particulares de contextos de violencia fruto de los conflictos y el narcotráfico, los cuales pueden ser determinantes en la participación electoral y que tímidamente han sido mencionados por la literatura.

En el tema de la estabilidad, los estudios muestran que los países con democracias establecidas y consolidadas presentan niveles inferiores de participación en relación con aquellos que corresponden a democracias nuevas; no obstante, algunos estudios ofrecen evidencias en otro sentido (O`Donnell y Schmitter, 1986 citado en Kostadinova y Power, 2007; Fornos, Power y Garand, 2004). En cuanto a la calidad de la democracia y su relación con la concurrencia a las urnas, buena parte de la literatura se vale de fenómenos como la corrupción (Stockemer, LaMontagne y Scruggs, 2012) y el clientelismo (Monroe, 1977) para ofrecer explicaciones a la pregunta que guía este artículo. 
Por otra parte, las investigaciones que estudian la violencia y su incidencia en la participación electoral explican que el comportamiento de los votantes se determina por criterios de seguridad y autoprotección. En ese sentido, en contextos de significativa conflictividad, la asistencia a las urnas se ve restringida en la medida en la que violencia genera emociones de angustia y temor en la población, lo que inhibe el comportamiento en lo público y fragmenta el tejido social; además, la falta de seguridad causada por el accionar de los actores ilegales conlleva a que las campañas electorales se alejen de la plaza pública, lo que incide en la información que pueden recibir los ciudadanos (Fernández y Aguilera, 2002; Trelles y Carreras 2012; Hoyos, 2009; Horbath, 2004).

\section{Consideraciones finales}

La revisión de los estudios sobre el comportamiento electoral demuestra que la literatura explora distintas dimensiones o enfoques que han contribuido significativamente con la respuesta a la pregunta de por qué se asiste a las urnas, más que limitarse a las explicaciones clásicas relacionadas con los factores socioeconómicos, psicológicos y racionales. De manera más específica, se evidencia que variables como la fatiga electoral, la proporcionalidad del sistema, el número de partidos, el tamaño de la población, lo reñido de las elecciones, entre otras, se configuran como puntos de referencia fundamentales para comprender el fenómeno.

Además, este ejercicio demuestra que no es suficiente abordar la pregunta por el comportamiento del elector a partir de un conjunto de variables separadas, como regularmente se han agrupado. Esto significa que una comprensión abarcadora del objeto de estudio conlleva a analizar la decisión del elector como un entramado donde confluyen variables, tanto de corte general como individual, cuyos mecanismos explicativos no pueden entenderse aisladamente, tal como se evidenció a lo largo del texto.

Finalmente, esta revisión permite evidenciar que el camino por recorrer es largo debido a que los métodos usados aún no permiten identificar variables que ayuden a entender de manera completa este problema, pues los mecanismos causales expuestos terminan siendo contradictorios para un mismo conjunto de variables en diferentes contextos. De tal manera, próximos estudios deberán centrar la atención en lograr descubrir dichos mecanismos teniendo en cuenta el contexto de los casos estudiados, así como variables subjetivas individuales que ayuden a entender procesos de identificación y desafección más allá de los sellos partidistas. 
Estudios sobre comportamiento electoral, ¿qué explica la participación en las urnas?...

\section{Referencias bibliográficas}

1. Andersen, Jørgen; Fiva, Jon \& Natavik, Gisle. (2014). Voting When The Stakes are High. CAMP Working Papers Series, 8, pp. 1-41. https://doi.org/10.1016/j. jpubeco.2013.10.003

2. Bedoya Marulanda, John Fredy. (2015). Los estudios del comportamiento del elector en América Latina: un análisis bibliográfico de los casos de México, Argentina, Chile y Colombia, 2000-2010. Estudios Políticos, 46, pp. 197-219.

3. Bellettini, Giorgio; Berti, Carlotta \& Monfardini, Chiara. (2014). Socio-Economic Heterogenety and Electoral Turnout: An Aggregate Analysis with Precint-Level Data. Workin Paper, 4999, pp. 1-26.

4. Ben-Bassat, Avi \& Dahan, Momi. (2012). Social Identity and Voting Behavior. Public Choice, 151, pp. 193-214. https://doi.org/10.1007/s11127-010-9742-2

5. Blais, André. (2006). What Affects Voter Turnout? Annual Review of Political Science, 9, pp. 111-125. https://doi.org/10.1146/annurev.polisci.9.070204.105121

6. Blais, André; Anduiza, Eva \& Gallego, Aina. (2011). Decentralization and Voter Turnout. Environment and Planning: Government and Policy, 29, pp. 297-320. https://doi.org/10.1068/c1015r

7. Blais, André; Singh, Shane \& Dumitrescu, Delia. (2014). Political Institutions, Perceptions of Representation, and the Turnout Decision. In: Thomassen, Jacques (ed.). Elections and Democracy: Representation and Accountability (pp. 99-112). New York: Oxford University. https://doi.org/10.1093/acprof:oso/9780198716334.003.0006

8. Boulding, Carew \& Brown, David. (2013). Do Political Parties Matter for Turnout? Number of Parties, Electoral Rules and Local Elections in Brazil and Bolivia. Party Politics, 21 (3), pp. 404-416. https://doi.org/10.1177/1354068813475496

9. Boyd, Richard. (1986). Elections Calendars and Voter Turnout. American Politics Quarterly, 14 (1-2), pp. 89-104. https://doi.org/10.1177/1532673X8601400106

10. Breux, Sandra, Couture, Jérôme \& Goodman, Nicole. (2016). Fewer Voters, Higher Stakes? The Applicability of Rational Choice for Voter Turnout. Environment and Planning: Government and Policy, 35 (6), pp. 990-1009.

11. Campbell, Angus; Converse, Philip; Miller, Warren \& Stokes, Donald. (1980). The American Voter. Nueva York: Wiley.

12. Cancela, João \& Geys, Benny. (2016). Explaining Voter Turnout: A MetaAnalysis of National and Subnational Elections. Electoral Studies, 42, pp. 264-275. https://doi.org/10.1016/j.electstud.2016.03.005

13. Caren, Neal. (2007). Big City, Big Turnout? Electoral Participation In American Cities. Journal Urban Affairs, 29 (1), pp. 31-46. https://doi.org/10.1111/j.14679906.2007.00321.x

14. Contreras, Gonzalo y Navia, Patricio. (2013). Diferencias generacionales en la participación electoral en Chile, 1988-2010. Revista de Ciencia Política, 33 (2), pp. 419-441. https://doi.org/10.4067/S0718-090X2013000200001 
15. Dalton, Russell \& Anderson, Christopher. (2010). Citizens, Context and Choice. How Context Shapes Citizen's Electoral Choices. Oxford: Oxford University. https://doi.org/10.1093/acprof:oso/9780199599233.001.0001

16. Delgado, Irene. (1998). El comportamiento electoral en los municipios rurales: una aproximación desde las elecciones municipales de 1995. Agricultura y Sociedad, 86, pp. 13-32.

17. Downs, Anthony. (1957). An Economic Theory of Democracy. Nueva York: Harper and Row.

18. Edlin, Aaron, Gelman, Andrew \& Kaplan, Noah. (2007). Voting as Rational Choice, Why and How People Vote to Improve their Well-being of Others. Rationality and Society, 19 (3), pp. 293-314. https://doi.org/10.1177/1043463107077384

19. Fernández, Lya y Aguilera, Armando. (2002). Violencia y elecciones en Santander. Reflexión Política, 8, pp. 192-199.

20. Fornos, Carolina; Power, Timothy \& Garand, James. (2004) Explaining Voter Turnout in Latin America. Comparative Political Studies, 37 (8), pp. 909-940. https:// doi.org/10.1177/0010414004267981

21. Freitag, Markus. (2010). Structure versus Culture: A Comparative Study of the Influence of Political Institutions and Cultural Modernization Factor on Voter Turnout in Swiss Sub-national Parlamentary Elections. International Political Science Review, 31 (4), pp. 428-448. https://doi.org/10.1177/0192512110371709

22. Funk, Patricia. (2008). Social Incentives and Voter Turnout: Evidence from the

[196 ] Swiss Mail Ballot System. Journal of Economic European Association, 8 (5), pp. 10771103. https://doi.org/10.1111/j.1542-4774.2010.tb00548.x

23. Gaarsted, Annie. (2002). Size and Electoral Participation in Local Elections. Environment and Planning. Government and Policy, 20, pp. 853-869. https://doi. org/10.1068/c0228

24. Galais, Carol \& Blais, André. (2015). Do People Feel More of a Duty to Vote in Some Elections? West European Politics, 39 (4), pp. 1-10.

25. Geys, Benny. (2006). Explaining Voter Turnout: A Review of Aggregatelevel Research. Electoral Studies, 25, pp. 637-663. https://doi.org/10.1016/j. electstud.2005.09.002

26. Gofman, Bernard \& Selb, Peter. (2010). Turnout and the (Effective) Number of Parties at the National and District Levels: A Puzzle-solving Approach. Party Politics, 17 (1), pp. 93-117. https://doi.org/10.1177/1354068810365506

27. Hajnal, Zoltan \& Lewis, Paul. (2003). Municipal Institutions and Voter Turnout in Local Elections. Urban Affairs Review, 38 (5), pp. 645-668. https://doi. org/10.1177/1078087403038005002

28. Henderson, Ailsa \& McEwen, Nicola. (2010). A Comparative Analysis of Voter Turnout in Regional Elections. Electoral Studies, 29, pp. 405-416. https://doi. org/10.1016/j.electstud.2010.03.012

29. Horbath, Jorge Enrique. (2004). Pobreza y elecciones en Colombia: Algunos hallazgos para reflexionar. Espiral, 10 (29), pp. 199-235. 
Estudios sobre comportamiento electoral, ¿qué explica la participación en las urnas?...

30. Hoyos, Diana. (2009). Dinámicas político electorales en zonas de influencia paramilitar. Análisis de la competencia electoral y la participación electoral. Análisis Político, 6, pp. 13-32.

31. Jackman, Robert \& Miller, Ross. (1995). Voter Turnout in the Industrial Democracies during the 1980s. Comparative Political Studies, 27 (4), pp. 467-492. https://doi.org/10.1177/0010414095027004001

32. Jácome, Javier. (2013). Patrones espaciales en las elecciones locales del 2007 en Bogotá, Colombia. Cuadernos de Geografía, 22 (1), pp. 141 -168. https://doi. org/10.15446/rcdg.v22n1.28612

33. Kaniovski, Serguei \& Mueller, Dennis. (2006). Community Size, Heterogeneity and Voter Turnouts. Public Choice, 129, pp. 399-415. https://doi.org/10.1007/s11127006-9063-7

34. Kostadinova, Tatiana \& Power, Timothy. (2007). Does Democratization Depress participation? Voter turnout in Latinamerican and Easterns Europe Transitional Democracies. Political Research Quarterly, 60 (3), pp. 363-377. https:// doi.org/10.1177/1065912907304154

35. Kuenzi, Michelle \& Lambrihgt, Gina. (2007). Voter Turnout in Africa's Multiparty Regimes. Comparative Political Studies, 40 (6), pp. 665-690. https://doi. org/10.1177/0010414006288969

36. Lago, Ignacio \& Costa, Marina. (2014). Partisan Turnout Bias and Distric Magnitud. Electoral Studies, 35, pp. 150-158. https://doi.org/10.1016/j. electstud.2014.06.001

37. Lago, Ignacio; Bermúdez, Sandra; Guinjoan, Marc \& Simón, Pablo. (2014). Turnout and Fractionalization. GEN Working Paper. Universidad de Vigo, pp. 1-31.

38. Lazarsfeld, Paul; Berelson, Bernard \& Gaudet, Hazel. (1968). The People's Choice: How the Voter Makes up His Mind in a Presidential Campaign (3ra ed.). Nueva York: Columbia University.

39. Lefevere, Jonas \& Van Aelst, Peter. (2014). First-order, Second-order or Third-rate? A Comparison of Turnout in European, Local and National Elections in the Netherlands. Electoral Studies, 35, pp. 159-170. https://doi.org/10.1016/j. electstud.2014.06.005

40. Mackena, Bernardo. (2015). Composición del electorado en elecciones con voto obligatorio y voluntario: un estudio cuasi experimental de la participación electoral en Chile. Revista Latinoamericana de Opinión Pública, 5, pp. 49-98.

41. Magalhães, Pedro; Segatti, Paolo \& Shi, Tianjian. (2015). Mobilization, Informal Networks and the Social Contexts of Turnout. In: Gunther, Richard; Beck, Paul Allen; Magalhães, Pedro \& Moreno, Alejandro (eds.). Voting in Old and New Democracies (pp. 64-98). New York: Routledge. https://doi.org/10.4324/9781315691558-3

42. Monroe, Alan. (1977). Urbanism and Voter Turnout: A Note on Some Unexpected Findings. American Journal of Political Science, 21 (1), pp. 71-78. https:// doi.org/10.2307/2110448 
43. Morlan, Robert. (1984). Municipal vs. National Election Voter Turnout: Europe and the United States. Political Science Quarterly, 99 (3), pp. 457-470. https:// doi.org/10.2307/2149943

44. Moya, Octaviano. (2007). Un nuevo modelo de decisión electoral: el comportamiento electoral en las elecciones de 2006 en México. Temas y Debates, 13, pp. 1-23.

45. Nownes, Anthony. (1992). Primaries, General Elections and Voter Turnout. A Multinomial Logit Model of the Decision to Vote. American Politics Quartely, 20 (2), pp. 205-226. https://doi.org/10.1177/1532673X9202000204

46. Ortega, Carmen, y Trujillo, José. (2013). La influencia del tamaño de las localidades sobre la participación electoral: un análisis contextual de las elecciones locales de 2011 en Andalucía. Revista de Investigaciones Políticas y Sociológicas, 12 (1), pp. 71-91.

47. Pérez Baralt, Carmen. (2006). Enfoques teórico-metodológicos en el estudio de la participación electoral. Cuestiones políticas, 37, pp. 74-93.

48. Riker, William H. \& Ordeshook, Peter C. (1968). A Theory of the Calculus of Voting. American Political Science Review, 62 (1), pp. 25-42. https://doi. org/10.2307/1953324

49. Sørensen, Rune J. (2016). The Impact of State Television on Voter Turnout. The British Journal of Political Science, pp. 1-22. https://doi.org/10.1017/ S000712341600048X

[198] 50. Stockemer, Daniel; LaMontagne, Bernadette \& Scruggs, Lyle. (2012). Bribes and Ballots. The Impact of Corruption on Voter Turnout in Democracies. International PoliticalScienceReview, 34(1), pp.74-90.https://doi.org/10.1177/0192512111419824

51. Tavares, António \& Carr, Jered. (2012). So Close, yet so Far Away? The Effects of City Size, Density and Growth on Local Civic Participation. Journal Of Urban Affairs, 35 (3), pp. 283-302. https://doi.org/10.1111/j.1467-9906.2012.00638.x

52. Trelles, Alejandro \& Carreras, Miguel. (2012). Bullets and Votes: Violence and Electoral Participation in Mexico. Journal of Politics in Latin America, 2, pp. 89-124.

53. Smets, Kaat \& Van Ham, Carolien. (2013). The Embarrassment of Riches? A Meta-Analysis of Individual-Level Research on Voter Turnout. Electoral Studies, 32 (2), pp. 344-359. https://doi.org/10.1016/j.electstud.2012.12.006

54. Soto, Irvin y Cortez, Willy. (2014). Determinantes de la participación electoral en México. Estudios Sociológicos, 32 (95), pp. 323-353.

55. Wang, Ching-Hsing. (2013). Why Do People Vote? Rationality or Emotion. International Political Science Review, 34 (5), pp. 483-501. https://doi. org/10.1177/0192512113490365

56. Zimmer, Troy A. (1983). Community and Communality in Voting Participation. Sociological Perspectives, 26 (2), pp. 185-199. https://doi.org/10.2307/1389090 